$\underline{\text { Original Article }}$

\title{
Decreased CD10 Expression in the Bone Marrow Neutrophils of HIV Positive Patients
}

Annemarie van de Vyver ${ }^{1}$, Eluned Delport ${ }^{2}$ and Adele Visser ${ }^{3}$

${ }^{1}$ Department Internal Medicine, Kalafong Hospital, University of Pretoria, ${ }^{2}$ Department Internal Medicine, Steve Biko Academic Hospital, University of Pretoria, ${ }^{3}$ Department Clinical Pathology, University of Pretoria, National, Health Laboratory Services.

Correspondence to: Annemarie van de Vyver, Department Internal Medicine, Kalafong Hospital, University of Pretoria, P O Box 2786, Faerie Glen, 0043 Pretoria. Telephone +2784 661 1822, Fax +2786 513 9469. E-mail: annievdvyver@gmail.com

Competing interests: The author have declared that no competing interests exist.

Published: November 15, 2010

Received: October 21, 2010

Accepted: November 05, 2010

Mediterr J Hematol Infect Dis 2010, 2(3): e2010032, DOI 10.4084/MJHID.2010.032

This article is available from: http://www.mjhid.org/article/view/6473

This is an Open Access article distributed under the terms of the Creative Commons Attribution License (http://creativecommons.org/licenses/by/2.0), which permits unrestricted use, distribution, and reproduction in any medium, provided the original work is properly cited

\begin{abstract}
Background: HIV-1 infection is associated with various quantitative and qualitative changes in haemopoietic cells. Clear distinction between primary myelodysplastic syndrome (MDS) and secondary dysplasia may not always be possible. Adjunctive analyses used in the diagnosis of MDS include cytogenetics and flow cytometry (FCM). Much focus has been placed on establishing FCM guidelines aiding in the diagnosis of MDS, and to distinguish this condition from secondary dysplastic changes. One of the parameters often cited is the CD10 expression on the granulocyte population, as this marker denotes granulocytic maturation. Aims: To determine the expression level of CD10 on granulocytes in HIV positive patients. Methods: In total, $117 \mathrm{HIV}-1$ positive and $29 \mathrm{HIV}-1$ negative patients were included in this study. Bone marrow aspirate samples were evaluated in terms of morphological abnormality as well as CD10 expression on the granulocytic population.

Results: The average CD10 expression among the HIV-1 positive patients were markedly reduced, at $18.4 \%$, and 113 patients $(96.6 \%)$ of these patients had expression levels below $50 \%$.

Discussion: Disease conditions causing secondary dysplasia, especially HIV-1 infection, is associated with a marked reduction in CD10 expression on the granulocyte population independent from the presence of myelodysplastic features. This marker is therefore of doubtful significance as a diagnostic tool in distinguishing between primary and secondary dysplasia.
\end{abstract}

Introduction: Infection with the Human Immunodeficiency Virus (HIV-1) is frequently 
associated with haematological abnormalities, ${ }^{1,2}$ including cytopaenias ${ }^{3}$ and dysplastic features., The aetiology for these changes are often multifactorial $^{6}$ including features of peripheral destruction, ${ }^{3}$ bone marrow suppression due to drugs, ${ }^{7}$ opportunistic infection ${ }^{8}$ or direct effect of HIV-1 on bone marrow precursor cells. ${ }^{9,10}$ HIV-1 infection has also been directly associated with myelosuppression with various possible underlying mechanisms postulated, including direct infection of haemopoietic progenitor cells, ${ }^{26,1}$ stromal cell functional impairment, toxic effects of HIV-1 proteins and alterations in the local cytokine milieu. ${ }^{1}$ The presence of dysplastic haemopoiesis in the bone marrows of HIV-1 positive patients have been well described, ${ }^{23}$ however, reports on MDS in HIV-1 positive patients are rare. ${ }^{24,23}$ Superficially these dysplastic changes can be morphologically similar to the dysplasia seen in primary myelodysplasia. ${ }^{30}$

The diagnosis of MDS calls for multidisciplinary evaluation of morphological, haematological and cytogenetic findings. ${ }^{4}$ As the more sophisticated investigations like cytogenetics yields aberrant findings in only about 20 to $70 \%$ of cases, ${ }^{11,12}$ novel methods of diagnosis are constantly evaluated. Recently, more emphasis has been placed the use of flow cytometry (FCM) in the diagnostic work-up of patients with MDS. ${ }^{27,20}$ Decreased or absent CD10 expression in the granulocyte series has been associated with MDS, and has been cited to occur in as much as $45 \%$ of MDS cases. ${ }^{16}$ Use of this marker has been proposed as a criterion in either diagnostic scoring systems ${ }^{4}$ or as supportive diagnostic criteria. ${ }^{27,16}$

However, expression levels of CD10 have not been evaluated in various other conditions, including known causes of secondary dysplasia, particularly HIV-1 infection. ${ }^{15}$ A recent publication from the European LeukemiaNet Working Conference $^{16}$ re-emphasized use of various parameters in the evaluation of patients with MDS, in an attempt to establish consensus guidelines in the diagnosis of MDS. For this reason, it remains imperative to fully understand the effect of various conditions on CD10 expression on the granulocyte population, as this may impact on future diagnostic criteria.

This is the first study to the authors' knowledge to investigate CD10 expression in granulocytic cells on bone marrow aspirate samples from HIV-1 positive patients with non-neoplastic haematological disorders.

\section{Materials and Methods:}

Patient Population: In total, 117 HIV-1 positive and 29 HIV-1 negative patients were included in this study. None of the HIV-1 positive patients had been initiated on highly active antiretroviral therapy (HAART) at the time of investigation and were in varying stages of HIV-1 disease, as reflected by CD4 counts. The presence of concurrent opportunistic infections was not known. Exclusion criteria included 1) patients younger than 12 years of age, 2) a diagnosis of any haematological neoplasm and 3) HIV-1 negative test (in the negative control group) performed more than 12 months from bone marrow examination. Flow cytometric data as performed on bone marrow aspirates were collected on all cases. None of the HIV-1 negative control group was considered to have primary myelodysplasia based on morphology or karyotyping.

Bone Marrow Morphology: Of the 117 samples included, only 70 included sufficient smear samples for evaluation of morphological features. The iron stain was used to evaluate the presence of sideroblasts, and quantified as low (less than 20\%), normal (20 to $30 \%$ ) or increased (more than $30 \%$ ), as well as the presence of pathologically overloaded forms.

Flow Cytometric Analysis: All bone marrow specimens were collected in ethylene-diaminetetraacetic acid (EDTA) or sodium-heparin tubes and processed within 24 hours. The white blood cell (WBC) count was determined using FLOWCOUNT, Beckman-Coulter (Miami, USA) and corrected to within the reference range (4-10 $\left.\mathrm{x} 10^{9} / \mathrm{L}\right)$ by either dilution with RPMI or buffy coat harvesting by density gradient extraction. A total volume of $100 \mu \mathrm{l}$ of the bone marrow specimen was mixed with $2 \mathrm{ml}$ of ammonium chloride to lyse red blood cells. The specimen was incubated for 10 minutes and centrifuged for 10 minute at 2000rpm. Viability was subsequently determined by using propidium iodide. Only specimens with a value above $75 \%$ viability were used.

According to our laboratory's standard operating procedure, $10 \mu \mathrm{l}$ of CD10 monoclonal antibody was added to $100 \mu 1$ of the bone marrow. Phycoerythrin (PE)-CD10 by Beckman-Coulter (Miami, USA) was used. Three colour flow was performed with the Cytomics FC500 flow 

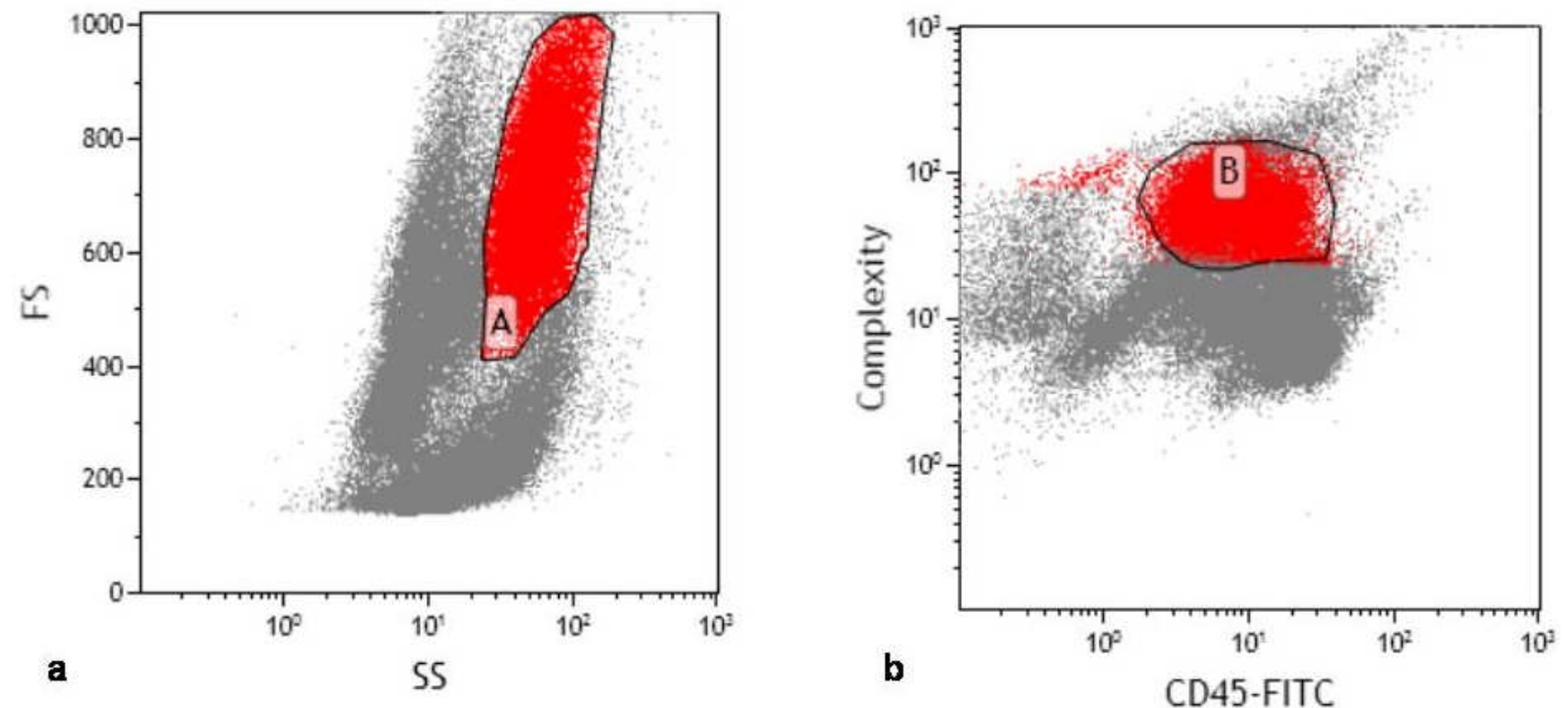

Figure 1: Granulocytes defined based on a. Side scatter (SS) versus Forward Scatter (FS) (population A) and b. CD45 versus SS (population B).
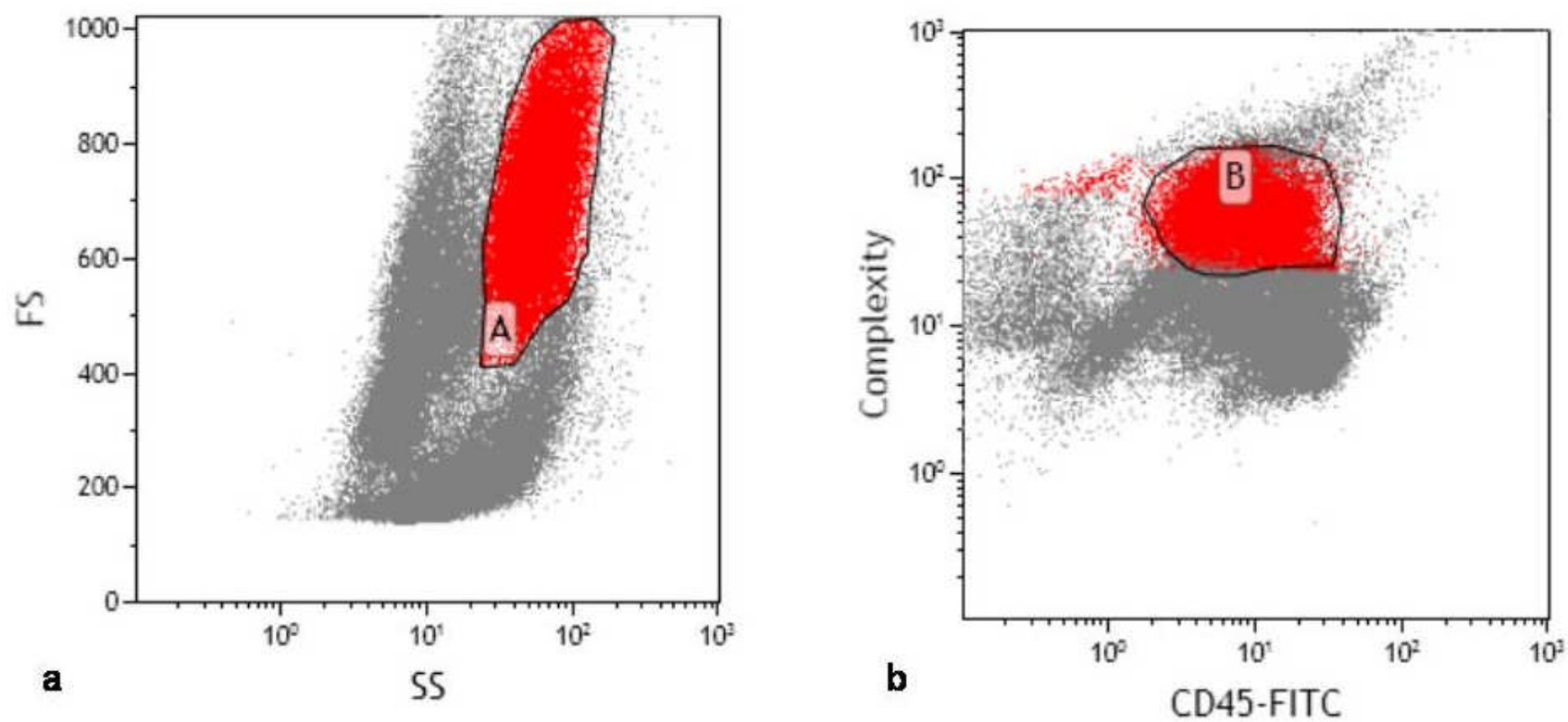

Figure 2: CD10 expression evaluated by a. CD5 FITC versus CD10-PE and b. CD10-PE versus CD19-ECD. Both sets were gated on population $\mathrm{A}$ as defined in figure 1, identified as granulocytes. Two discrete populations can be demonstrated in each defined as either CD10 positive (population $\mathrm{C}$ and I) or negative (populations $\mathrm{D}$ and $\mathrm{H}$ ).

cytometer or XL-MCL, Beckman-Coulter (Miami, USA) equipped with a $15-\mathrm{mW}$ argon laser (excitation at $488 \mathrm{~mm}$ ). Antibody panel used included CD5-Fluorescein isothiocyanate (FITC) vs CD10-PE vs CD19-PE-Texas red (ECD). Granulocytes were defined using side scatter versus forward scatter (Figure 1a) and CD45 (dim) versus side scatter (Figure 1b). CD10 expression was determined within this population. Compensation within this gate was performed by also incubating cells with appropriate fluorochrome-coupled isotype control antibodies.The CD10 expression was quantified from the CD5-FITC versus CD10-
$\mathrm{PE}$ analysis (Figure 2a) and verified by comparison to CD10-PE versus CD19-ECD (Figure 2b). CD10 $\mathrm{PE}$ was used to estimate the total CD10 expression of the granulocyte population. Standardization of instrument electronics was done using Flow Check Fluorospheres calibration beads by BeckmanCoulter (Miami, USA) according to the manufacturer's recommendations. Compensation was adjusted using FITC-CD4/PE-CD8/PerCPCD3-stained cells. Isotypic controls were used as negative controls to eliminate non-specific binding. In each cell preparation, 15 to 30 thousand total events were collected. Data were analyzed with 
Table 1. Baseline demographic and results of study population Study population

\begin{tabular}{ccc} 
& \multicolumn{2}{c}{$n=148$} \\
\cline { 2 - 3 } Variable & HIV-1 positive & HIV-1 negative \\
patients & patients \\
$n=117$ & $\mathrm{n}=29$ \\
\hline
\end{tabular}

\begin{tabular}{lcc} 
Age (in years) & $37(23-77)$ & $42(12-85)$ \\
$\begin{array}{l}\text { Average CD4 } \\
\text { count }\end{array}$ & $129 \pm 151$ & N/A \\
$\begin{array}{l}\text { Average CD10\% } \\
\text { expression* }\end{array}$ & $18.40 \%$ & $37.10 \%$ \\
\hline $\begin{array}{l}\text { Number of } \\
\text { subjects with } \\
\text { CD10\% } \\
\text { expression* }<\mathbf{5 0 \%}\end{array}$ & $133(96.6 \%)$ & $22(75.9 \%)$ \\
\hline
\end{tabular}

* CD10\% expression on granulocyte population

CXP cytometer software, Beckman-Coulter (Miami, USA) gating the total leukocyte population.

\section{Results:}

Patient population: Ages for the patients varied from 23 to 77 years (mean age of 37 years) for the HIV-1 positive patients and 12 to 85 years (mean age of 42 years) for the HIV-1 negative patient group (Table 1). Although not HIV-1 infected, the latter group had various underlying medical conditions which is associated with secondary dyplasia. This included 7 cases with haematinic deficiencies, be it iron, folate, vitamin B12 or a combination of these. Also included were 4 patients with systemic sepsis, 4 with auto-immune disease, 3 each with chronic renal failure and chronic inflammatory diseases, 2 with thyroid disease and 1 with liver cirrhosis. Three of the patients were admitted with haematological disorders involving either platelets or red cells.

Bone Marrow Morphology: Various dysplastic features could be noted in all cell lines (Table 2). Of note was the presence of trilineage dysplasia in one third of the patients, and bilineage dysplasia in another third. Dysplasia was noted in only one lineage in $26 \%$ of cases and no dysplastic features were noted in $9 \%$. Blast counts were not increased in any of the HIV-1 positive patients, and monocytosis was a rare finding. Although dysplasia was a frequent finding, it could not be demonstrated in more than $10 \%$ of the myeloid lineage, and therefore did not meet the WHO diagnostic criteria for primary myelodysplastic syndrome.

Flow Cytometric Analysis: In the HIV-1 positive study group, the average CD10 expression on granulocytes were $18.4 \%$ (SD of 15.83) compared to the HIV-1 negative group with levels of $37.1 \%$ (SD 21.2) (Figure 3). In the HIV-1 positive group $113 / 117(96.6 \%)$ patients had a total CD10 expression of less than $50 \%$, in contrast to $22 / 29$ $(75.9 \%)$ the HIV-1 negative group (table 1)( $\mathrm{p}<0.0001)$. A left shift in the granulocyte lineage could be demonstrated in $<30 \%$ of cases. Exclusion of these left-shifted cases did not have a statistically significant impact on the data, increasing the average CD10 expression to $18.5 \%$. No correlation between CD10 granulocyte percentage and CD4 counts (as a marker for HIV-1 disease progression) could be demonstrated in the HIV-1 positive population $(\mathrm{p}>0.34)$.

Table 2. Dysplastic features in HIV-1 infected patients.

\begin{tabular}{|c|c|c|}
\hline & $\begin{array}{l}\text { Dysplastic Features } \\
n=70\end{array}$ & $\begin{array}{c}\text { Absolute } \\
\text { (percentage) }\end{array}$ \\
\hline \multirow{3}{*}{$\begin{array}{l}\text { Bone marrow } \\
\text { cellularity }\end{array}$} & Hypercellular & $31(44 \%)$ \\
\hline & Normocellular & $35(50 \%)$ \\
\hline & Hypocellular & $5(6 \%)$ \\
\hline \multirow{6}{*}{$\begin{array}{l}\text { Granulocytic } \\
\text { Lineage }\end{array}$} & Left shift & $21(30 \%)$ \\
\hline & Giant Metamyelocytes & $33(47 \%)$ \\
\hline & Monocytosis & $1(1 \%)$ \\
\hline & Hypogranularity & $3(4 \%)$ \\
\hline & Hyposegmentation* & $24(34 \%)$ \\
\hline & Hypersegmentation & $9(13 \%)$ \\
\hline \multirow{4}{*}{ Erythroid lineage } & Red cell dysplasia§ & $57(81 \%)$ \\
\hline & Macronormoblasts & $11(16 \%)$ \\
\hline & Megaloblasts & $25(36 \%)$ \\
\hline & $\begin{array}{l}\text { Multinuclear erythroid } \\
\text { cells }\end{array}$ & $14(20 \%)$ \\
\hline \multirow{2}{*}{$\begin{array}{l}\text { Megakaryocytic } \\
\text { lineage }\end{array}$} & Micromegakaryocytes & $4(6 \%)$ \\
\hline & $\begin{array}{l}\text { Atycal nuclear } \\
\text { configurations }\end{array}$ & $26(37 \%)$ \\
\hline \multirow{3}{*}{ Sideroblasts $(n=46)$} & Decreased & $35(22 \%)$ \\
\hline & Normal & $1(2 \%)$ \\
\hline & $\begin{array}{l}\text { Incresed/pathologically } \\
\text { overloaded }\end{array}$ & $10(76 \%)$ \\
\hline
\end{tabular}

*Hyposegmentation includes Pseudo-Pelger-Huet forms and ring forms

$\S$ Red cell Dysplasia includes uneven haemoglobinization, ragged cytoplasm and intercytoplasmic bridging 


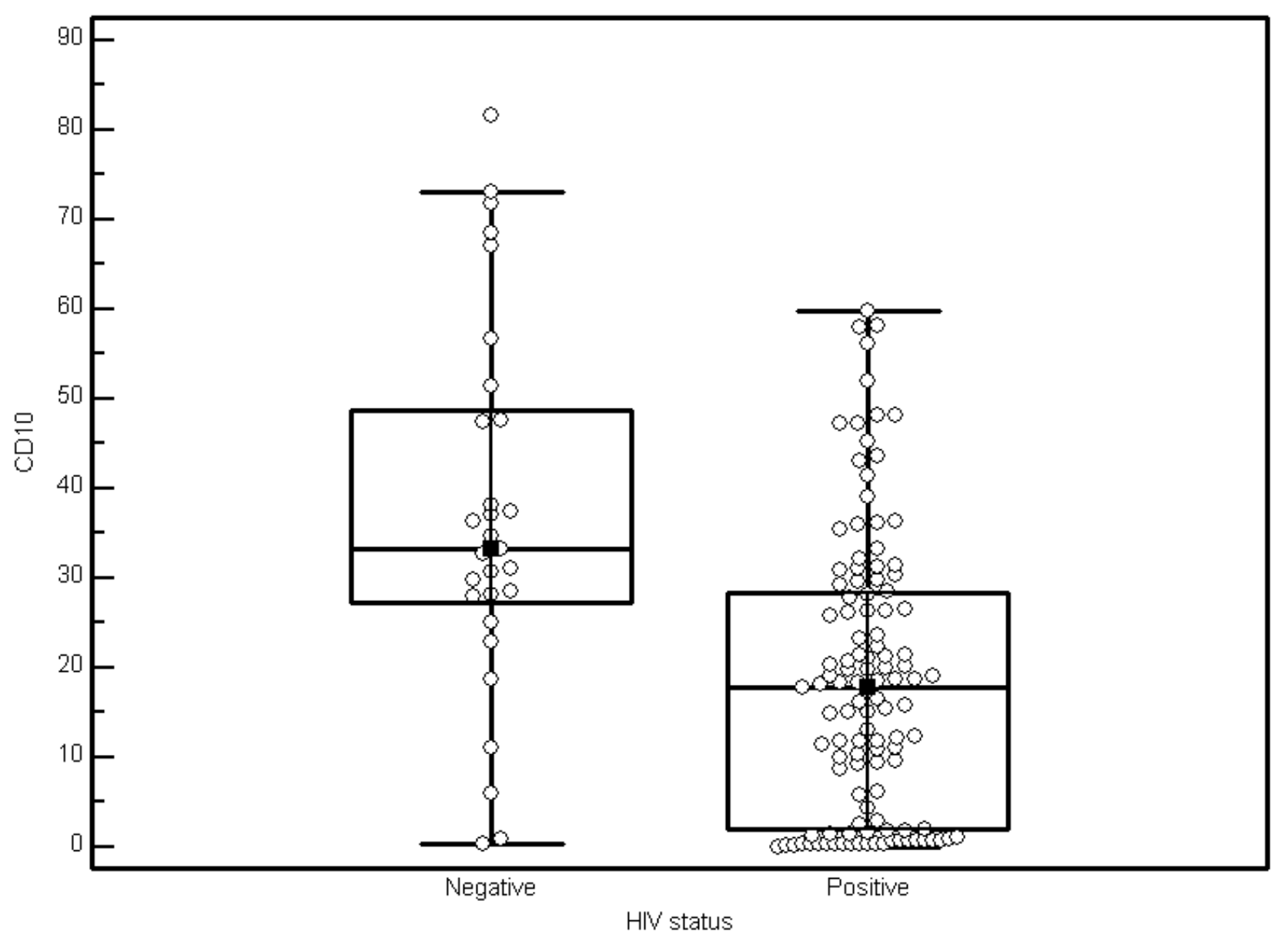

Figure 3. CD10 expression depicted as a Box and Whisker plot

Discussion: Changes in bone marrow morphology associated with HIV-1 infection has been evaluated by various authors. ${ }^{1,5,8,28}$ These studies showed that certain findings are common to both HIV-1 and MDS. These include increased bone marrow cellularity, left shift in granulocytes, presence of giant band cells and megaloblastoid changes in the erythroid lineage, consistent with the findings in the current study. Macrocytosis can also be present especially secondary to drugs administered in the treatment of HIV-1. Findings found in primary MDS, in contrast to HIV-1 dysplasia include an increase in blast cells, hypogranularity as well as hyper- or hyposegmentation of granulocytes and micromegakaryocytes with atypical lobulation, ${ }^{23}$ but the blast counts were not elevated. These finding were not in keeping with the current study, as hypogranularity, hyposegmentation, hypersegmentation and the dysplastic features in megakaryocytes were relatively common among our HIV-1 positive population. These findings have been cited as useful in delineating between these two conditions, however, changes may be subtle. Depending on the different subclasses of primary
MDS, degrees of dysplasia may also vary. ${ }^{23}$ Therefore, distinguishing morphologically between primary and secondary dysplasia can be very troublesome.

For all these reasons, establishing the underlying cause of dysplasia should be approached as a multidisciplinary work-up. Although considered diagnostic, cytogenetic analysis typically contributes to only $40 \%$ of cases, showing a variety of abnormalties. ${ }^{4}$ Consequently, scoring systems have been advocated for use in diagnosis to improve sensitivity and specificity of diagnosis. ${ }^{4,16}$ Various flow cytometric parameters have been evaluated as useful diagnostic indicators in MDS, including use of CD10 expression on granulocytic cells. ${ }^{21}$

Expression of CD10 has been examined as a marker of granulocyte maturation in various studies, ${ }^{12,14}$ as it is expressed by terminally differentiated neutrophils. ${ }^{25}$ Levels have been evaluated by numerous authors, showing reduced levels on granulocytes varying from 20 to $70 \%,{ }^{11}$ depending on the subtype of MDS. Secondary dysplastic changes can occur due to various 
conditions and should be excluded before the diagnosis of MDS can be considered. ${ }^{3}$ With specific relevance to HIV-1 positive patients, long term use of zidovudine (AZT) has been shown to induce dysplasia in mouse studies. ${ }^{2}$ However use of gancyclovir and trimethoprim-sulfamethoxaxole (standard prophylactic antibiotic used according to the South African guidelines) have not been shown to induce dysplasia in vivo. ${ }^{23}$ The HIV-1 negative group also included some patients with other causes of secondary dysplasia. ${ }^{31}$ This again correlates with the finding of reduced CD10 expression in this population as well, where almost $76 \%$ of patients had levels below $50 \%$. In light of these findings, decreased CD10 expression may correlate with myeloid dysplasia, not only in primary, but also secondary dysplastic conditions.

Decreased CD10 expression has been associated with increased susceptibility to infections, likely due to its role in neutrophil chemotaxis and coordination of the inflammatory response. ${ }^{22}$ This becomes clinically evident amongst neonates, patients with burns and those with systemic sepsis, all of which have been shown to have both reduced CD10 expression on their granulocytes, and a degree of immunosuppression. ${ }^{6}$ The clinical implications of the reduced CD10 expression noted in HIV-1 positive patients remain unknown.

The diagnostic utility of FCM has not been evaluated in HIV positive patient's bone marrow samples. The interrelationship between the presence of dysplasia and the effect on the function of the bone marrow has also not been studied. In this study, HIV-1 positive patients showed a significant reduction in CD10 expression, with $96.6 \%$ of the patients showing expression below 50\%. This calls into doubt the use of this marker as an adjunct in the diagnosis of MDS.

Conclusion: Flow cytometry is a useful adjunct in the diagnosis of MDS. Although a single antigen abnormality cannot be used in isolation to diagnose MDS, it remains of paramount importance to evaluate the merit of each antigen within clinical context. Utility of specific markers should however be carefully evaluated as causes of secondary dysplasia may also elicit similar immunophenotypic abnormalities, currently advocated as markers specific for primary myelodysplasia. A full understanding needs to be gained on immunophenotypic changes associated with various stages of HIV-1 infection, to so facilitate use of this diagnostic adjunct in this patient population. The impact of these aberrancies needs to be clinically correlated with the degree of immunological dysfunction.

Acknowledgements: The authors would like to express their appreciation to Dr Van Biljon for assisting in data collection. We are further in dept to the Department Haematology, University of Pretoria, for their contribution to this work. Dr Visser's work is supported by the Discovery Foundation.

\section{References:}

1. Alexaki, A. and B. Wigdahl (2008). "HIV-1 infection of bone marrow hematopoietic progenitor cells and their role in trafficking and viral dissemination." PLoS Path 4(12): 1-12.

2. Baurmann, H., T. Schwarz, et al. (1992). "Acute parvovirus B19 infection mimicking myelodysplatic syndrome of the bone marrow." Ann Hematol 64: 43-45.

3. Bowen, D., D. Culligan, et al. (2003). "Guidelines for the diagnosis and therapy of adult myelodysplastic sydnromes." Br J Haematol 120: 187-200.

4. Cherian, S., J. Moore, et al. (2005). "Peripheral Blood MDS Score: A New Flow Cytometric Tool for the Diagnosis of Myelodysplastic Syndromes." Cytometry Part B 64B: 9-17.

5. Claster, S. (2002). "Biology of Anaemia: Differential Diagnosis and Treatment Options in Human Immunodeficiency Virus Infection." J Infect Dis 185(Suppl 2): S105-109.

6. Connelly, J., R. Skidgel, et al. (1985). "Neural endopeptidase 24.11 in human neutrophils: Cleavage of chemotactic peptide." Proc natl Acad Sci USA 82: 87378741 .

7. Costello, C. (1988). "Haematological anormalities in human immunodeficiency virus (HIV) disease." J Clin Pathol 41: 711-715.

8. Coyle, T. (1997). "Hematologic complications of human immunodeficiency virus infection and the acquired

immunodeficiency syndrome." Med Clin North Am 81: 449 470 .

9. Daloutsi, V., U. Kohlmeyer, et al. (1994). "Comparison of bone marrow and hematologic findings in patients with human immunodeficiency virus infection and those with myelodysplatic syndromes and infectious disease." A J Clin Pathol 101: 123-129.

10. Gallicchio, V., K. Tse, et al. (1996). "Suppression of hematopoietic support function is associated with overexpression of interleukin-4 and transforming growth factor-b1 in LP-BM5 murine-leukemia-virus-infected stromal cell lines." Acta Haematol 95: 204-212.

11. Greenberg, P., C. Cox, et al. (1997). "International scoring systems for evaluating prognosis in myelodysplastic syndromes." Blood 89: 2079-2088.

12. Hanson, C. (1997). "Myelodysplastic syndrome and the patient with unexplained cytopaenias." Am J Clin Pathol 107: 261-264.

13. Inoue, T., E. Cronkite, et al. (1997). "Lifetime treatment of mice with Azidothymidine (AZT) produces myelodysplasia." Leukemia 11 (Suppl 3): 123-127.

14. LeBien, T. and R. McCormack (1989). "The common acute lymphoblastic leukemia antigen (CD10) - emancipation from a functional enigma." Blood 73(3): 625-635.

15. Loken, M., A. v. d. Lossdrecht, et al. (2008). "Flow 
cytometry in myelodysplastic syndromes: Report from a working conference." Leuk Res 32: 5-1.

16. Loosdrecht, A. v. d., C. Alhan, et al. (2009). "Standardization of flow cytometry in myelodysplastic syndromes: report from the first European LeukemiaNet working conference on flow cytometry in myelodysplastic syndromes." Haematologica 94(8): 1124-1134.

17. Ludewig, B., H. Gelderblom, et al. (1996). "Transmission of HIV-1 from productively infected mature Langerhans cells to primary CD4+ $\mathrm{T}$ lymphocytes results in altered $\mathrm{T}$ cell responses with enhances production of IFN-gamma and IL10." Virology 215: 51-60.

18. Mir, N., C. Costello, et al. (1989). "HIV-disease and bone marrow changes: A study of 60 cases." Eur J Haematol 42(4): 339-343.

19. Moses, A., S. Williams, et al. (1996). "Human immunodeficiency virus infection of bone marrow endothelium reduces induction of stromal hematopoietic growth factors." Blood 87: 919-925.

20. Ogata, K., K. Nakamura, et al. (2002). "Clinical significance of phenotypic features of blasts in patients with myelodysplastic syndrome." Blood 100(3887-3896).

21. Ogata, K., M. D. Porta, et al. (2009). "Diagnostic utility of flow cytometry in low-grade myelodysplastic syndromes: a prospective validation study." Haematologica 94(8): 10661074.

22. Ohsaka, A., K. Saionji, et al. (1994). "Complement receptor type 1 (CR1) deficiency on neutrophils in myelodysplastic syndrome." Br J Haematol 88: 409-412.

23. Ryu, T., M. Ikeda, et al. (2001). "Myelodysplasia Associated with Acquired Immunodeficiency Syndrome." Internal Medicine 40: 795-801.

24. Schneider, D. and L. Picker (1985). "Myelodysplasia in the acquired immune deficiency syndrome." Am J Clin Pathol 84: $144-152$.

25. Shipp, M. and A. Look (1993). "Hemopoietic Differentiation Antigens That Are Membrane-Associated Enzymes: Cutting is the Key!" Blood 82(4): 1052-1070.

26. Steinberg, H., C. Crumpacker, et al. (1991). "In vitro suppression of normal human bone marrow progenitor cells by human immunodeficiency virus." J Virol 65: 1765-1769.

27. Stetler-Stevenson, M., D. Arthur, et al. (2001). "Diagnostic utility of flow cytometric immunophenotyping in myelodsyplastic syndromes." Blood 98: 979-987.

28. Tanaka, P., D. Hadad, et al. (2007). "Bone marrow biopsy in the diagnoses of infectious and non-infectious causes in patients with advanced HIV infection." J Infect 54(4): 362366.

29. Thiele, J., T. Zirbes, et al. (1996). "AIDS-related bone marrow lesions-myelodysplastic features or predominant inflammatory-reactive changes (HIV-myelopathy)? A comparative morphometric study by immunohistochemistry with special emphasis on apoptosis and PCNA-labelling." Anal Cell Pathol 11: 141-157.

30. Tripathi, A., R. Misra, et al. (2005). "Bone marrow abnormalities in HIV disease." J Assoc Physicians India 53: 705-710.

31. Westerman, D., D. Evans, et al. (1999). "Neutrophil hypersegmentation in iron deficiency anaemia: a case$\begin{array}{llll}\text { control study." } & \text { BJH } & \mathbf{1 0 7}(3) \text { : } & \text { 512-515. }\end{array}$ 